Acta Crystallographica Section E

Structure Reports

Online

ISSN 1600-5368

\section{3,5-Dinitrobenzyl methanesulfonate}

\section{Gul S. Khan, George R. Clark and David Barker*}

Chemistry Department, The University of Auckland, Private Bag 92019, Auckland, New Zealand

Correspondence e-mail: g.clark@auckland.ac.nz

Received 1 July 2008; accepted 6 July 2008

Key indicators: single-crystal $\mathrm{X}$-ray study; $T=89 \mathrm{~K}$; mean $\sigma(\mathrm{C}-\mathrm{C})=0.002 \AA ; R$ factor $=$ $0.033 ; w R$ factor $=0.088$; data-to-parameter ratio $=13.7$.

The title compound, $\mathrm{C}_{8} \mathrm{H}_{8} \mathrm{~N}_{2} \mathrm{O}_{7} \mathrm{~S}$, an intermediate in the synthesis of $N, N$-bis(2-hydroxyethyl)-3,5-dinitroaniline, exists as a discrete molecule; the nitro groups are twisted with respect to the aromatic system [dihedral angles $=17.0$ (1) and $\left.26.3(1)^{\circ}\right]$.

\section{Related literature}

For the utility of benzyl methanesulfonates in synthesis, see: Barker et al. (2008); Bretonniere et al. (2004); Oh et al. (2004); Schirok et al. (2005). For the incorporation of $N, N$-bis(2hydroxyethyl)benzylamines in macromolecular metal complexes, see: Crans \& Boukhobza (1998); Koizumi et al. (2005, 2007).

\section{Experimental}

Crystal data

$\mathrm{C}_{8} \mathrm{H}_{8} \mathrm{~N}_{2} \mathrm{O}_{7} \mathrm{~S}$

Monoclinic, $P 2_{1} / c$

$a=9.3549(5) \AA$

$b=8.7552(5) \AA$

$c=14.1526(8) \AA$

$\beta=107.430(1)^{\circ}$

$V=1105.91(11) \AA^{3}$

$Z=4$

Mo $K \alpha$ radiation

$\mu=0.32 \mathrm{~mm}^{-1}$

$T=89(1) \mathrm{K}$

$0.32 \times 0.14 \times 0.14 \mathrm{~mm}$

\section{Data collection}

Bruker SMART diffractometer

Absorption correction: multi-scan

(SADABS; Sheldrick, 1997)

$T_{\min }=0.799, T_{\max }=0.971$

6374 measured reflections 2233 independent reflections 1959 reflections with $I>2 \sigma(I)$ $R_{\text {int }}=0.019$

Refinement

$R\left[F^{2}>2 \sigma\left(F^{2}\right)\right]=0.033$

$w R\left(F^{2}\right)=0.088$

$S=1.06$

2233 reflections

163 parameters

$\mathrm{H}$-atom parameters constrained

$\Delta \rho_{\max }=0.28{\mathrm{e} \AA^{-3}}^{-3}$

$\Delta \rho_{\min }=-0.49{\mathrm{e} \AA^{-3}}^{-3}$

Data collection: SMART (Bruker, 1995); cell refinement: SAINT (Bruker, 1995); data reduction: $S A I N T$; $\operatorname{program}(\mathrm{s})$ used to solve structure: SHELXS97 (Sheldrick, 2008); program(s) used to refine structure: SHELXL97 (Sheldrick, 2008); molecular graphics: ORTEPIII (Burnett \& Johnson, 1996); software used to prepare material for publication: SHELXTL (Sheldrick, 2008).

The authors acknowledge financial support from the Higher Education Commission of Pakistan and the University of Auckland, New Zealand.

Supplementary data and figures for this paper are available from the IUCr electronic archives (Reference: NG2470).

\title{
References
}

Barker, D., Lehmann, A. L., Mai, A., Khan, G. S. \& Ng, E. (2008). Tetrahedron Lett. 49, 1660-1664.

Bretonniere, Y., Cann, M. J., Parker, D. \& Slater, R. (2004). Org. Biomol. Chem. 2, 1624-1632.

Bruker (1995). SMART and SAINT Bruker AXS Inc., Madison, Wisconsin, USA.

Burnett, M. N. \& Johnson, C. K. (1996). ORTEPIII. Report ORNL-6895. Oak Ridge National Laboratory, Tennessee, USA.

Crans, D. C. \& Boukhobza, I. (1998). J. Am. Chem. Soc. 120, 8069-8078.

Koizumi, S., Nihei, M., Nakano, M. \& Oshio, H. (2005). Inorg. Chem. 44, 12081210.

Koizumi, S., Nihei, M., Shiga, T., Nakano, M., Nojiri, H., Bircher, R., Waldmann, O., Ochsenbein, S. T., Guedel, H. U., Fernandez-Alonso, F. \& Oshio, H. (2007). Chem. Eur. J. 13, 8445-8453.

Oh, S.-J., Lee, K. C., Lee, S.-Y., Ryu, E. K., Saji, H., Choe, Y. S., Chi, D. Y., Kim, S. E., Lee, J. \& Kim, B.-T. (2004). Bioorg. Med. Chem. 12, 5505-5513.

Schirok, H., Alonso-Alija, C., Benet-Buchholz, J., Goeller, A. H., Grosser, R., Michels, M. \& Paulsen, H. (2005). J. Org. Chem. 70, 9463-9469.

Sheldrick, G. M. (1997). SADABS. Univ. of Göttingen, Germany.

Sheldrick, G. M. (2008). Acta Cryst. A64, 112-122. 


\section{supporting information}

Acta Cryst. (2008). E64, o1470 [doi:10.1107/S1600536808020850]

\section{3,5-Dinitrobenzyl methanesulfonate}

\section{Gul S. Khan, George R. Clark and David Barker}

\section{S1. Comment}

Benzylic methansulfonates are readily prepared from benzylic alcohols and are often more easily prepared and more stable than the corresponding benzylic halide (Barker et al., 2008). In particular benzylic methanesulfonates are useful for the preparation of $N, N$-bis(2-hydroxyethyl)benzylamines, which are nitrogen mustard precursors. The dual functionality of the two free hydroxyl groups along with a basic nitrogen have also seen $N, N$-bis(2-hydroxyethyl)benzylamines used in synthesis of numerous metal complexes including those containing vanadium (Crans \& Boukhobza, 1998), manganese (Koizumi et al., 2005, 2007) and iron (Koizumi et al., 2005). There are no hydrogen bonding or $\pi-\pi$ interactions in the crystal. The closest intermolecular contacts are $\mathrm{O} 3 \cdots \mathrm{N} 1$ of $2.83 \AA$, and a pair of O $\cdots \mathrm{O} 3.32 \AA$ contacts between sulfonate oxygen atoms.

\section{S2. Experimental}

To a solution of 3,5-dinitrobenzyl alcohol $(1.5 \mathrm{~g}, 7.57 \mathrm{mmol})$ and triethylamine $(1.58 \mathrm{ml}, 11.35 \mathrm{mmol})$ in dry THF (15 $\mathrm{ml})$ at $0^{\circ} \mathrm{C}$, under an atmosphere of nitrogen, was added dropwise a solution of methanesulfonyl chloride $(0.88 \mathrm{ml}, 11.35$ $\mathrm{mmol})$ in dry THF ( $5 \mathrm{ml}$ ) and the resulting solution stirred at room temperature for $3 \mathrm{~h}$. The solvent was removed in vacuo and the residue diluted with ethyl acetate $(150 \mathrm{ml})$, washed with brine $(50 \mathrm{ml})$, dried $\left(\mathrm{MgSO}_{4}\right)$ and the solvent removed in vacuo to afford the title compound ( $2 \mathrm{~g}, 96 \%)$ as a yellow solid which was recrystallized from ethyl acetate to give yellow crystals (m.p. 356-357 K) suitable for X-ray crystallography. IR vmax (NaCl)/cm ${ }^{-1} 3399,1627,1541,1458$, 1344. ${ }^{1} \mathrm{H}$ NMR (400 MHz, $\mathrm{CDCl}_{3}$, $\delta$, p.p.m.) $3.15\left(3 \mathrm{H}, \mathrm{s}, \mathrm{CH}_{3}\right), 5.40\left(2 \mathrm{H}, \mathrm{s}, \mathrm{CH}_{2} \mathrm{O}\right), 8.60(2 \mathrm{H}$, br s, $\mathrm{Ar}-\mathrm{H}), 9.05(1 \mathrm{H}$, br s, $\mathrm{Ar}-\mathrm{H}) . \delta_{\mathrm{C}}\left(100 \mathrm{MHz}, \mathrm{CDCl}_{3}, \delta\right.$, p.p.m.) $38.6\left(\mathrm{CH}_{3}, \mathrm{CH}_{3}\right), 67.4\left(\mathrm{CH}_{2}, \mathrm{CH}_{2} \mathrm{O}\right), 119.5(\mathrm{CH}, \mathrm{Ar}-\mathrm{C}), 128.2(\mathrm{CH}, \mathrm{Ar}-\mathrm{C})$, 138.6 (CH, Ar-C), 149.1 (quat., $\mathrm{Ar}-\mathrm{C}$ ). MS m/z (EI) 276 (M+1\%), 197 (100), 181 (42), 134 (20). HRMS (EI) Found $M^{+} 276.00489, \mathrm{C}_{8} \mathrm{H}_{8} \mathrm{~N}_{2} \mathrm{O}_{7} \mathrm{~S}$ requires 276.00522 .

\section{S3. Refinement}

Hydrogen atoms were placed in calculated positions and refined using the riding model [C- $-\mathrm{H} 0.93-0.97 \AA)$, with $U_{\text {iso }}(\mathrm{H})$ $=1.2$ or 1.5 times $U_{\mathrm{eq}}(\mathrm{C})$. 


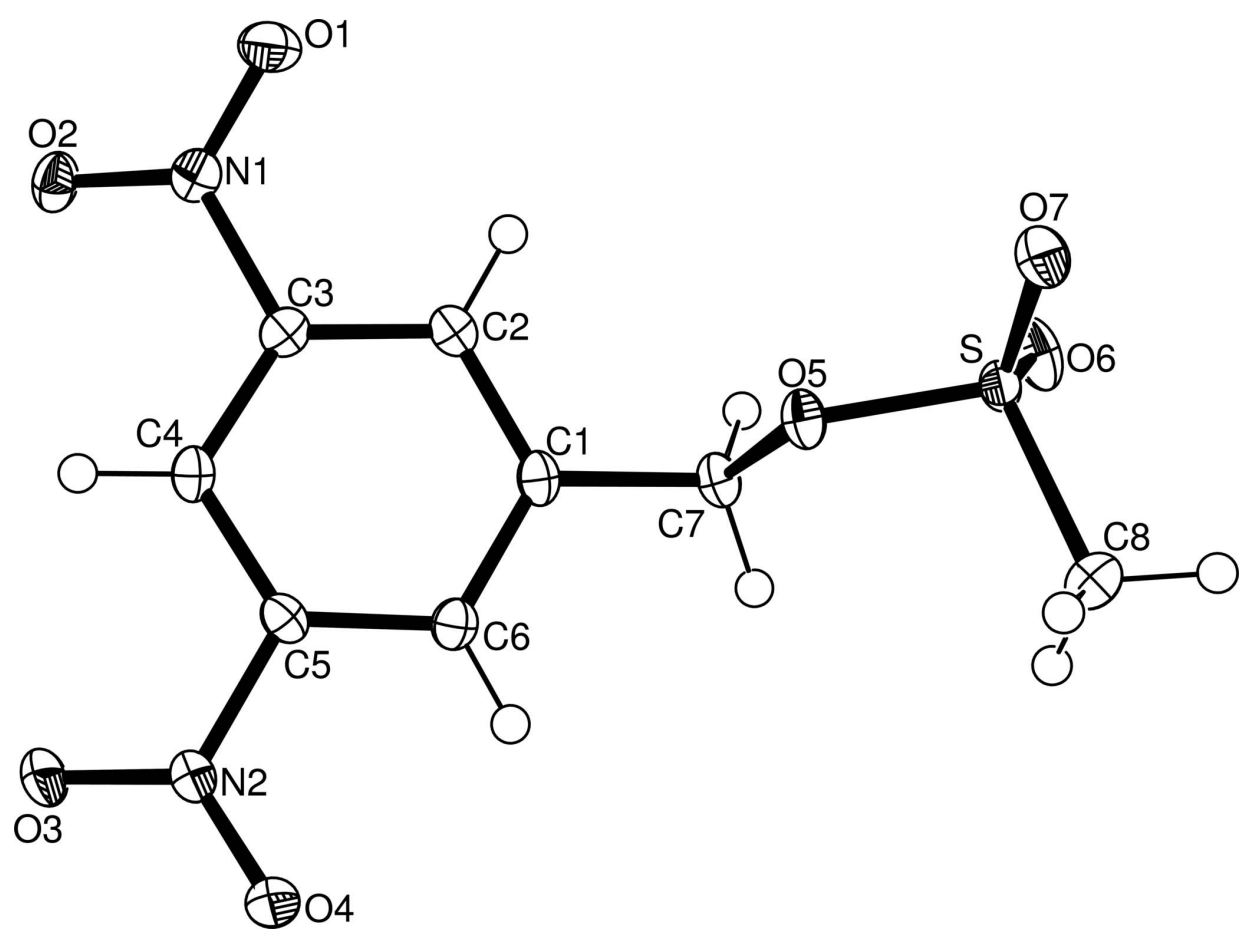

\section{Figure 1}

Structure showing 50\% probability displacement ellipsoids for non-hydrogen atoms and hydrogen atoms as arbitary spheres (Burnett \& Johnson, 1996).

\section{3,5-Dinitrobenzyl methanesulfonate}

\section{Crystal data}

\section{$\mathrm{C}_{8} \mathrm{H}_{8} \mathrm{~N}_{2} \mathrm{O}_{7} \mathrm{~S}$}

$M_{r}=276.22$

Monoclinic, $P 2{ }_{1} / c$

Hall symbol: -P $2 \mathrm{ybc}$

$a=9.3549$ (5) $\AA$

$b=8.7552(5) \AA$

$c=14.1526(8) \AA$

$\beta=107.430(1)^{\circ}$

$V=1105.91(11) \AA^{3}$

$Z=4$

\section{Data collection}

\section{Bruker SMART}

diffractometer

Radiation source: fine-focus sealed tube

Graphite monochromator

$\omega$ scans

Absorption correction: multi-scan

(SADABS; Sheldrick, 1997)

$T_{\min }=0.799, T_{\max }=0.971$
$F(000)=568$

$D_{\mathrm{x}}=1.659 \mathrm{Mg} \mathrm{m}^{-3}$

Mo $K \alpha$ radiation, $\lambda=0.71073 \AA$

Cell parameters from 4665 reflections

$\theta=2.3-26.4^{\circ}$

$\mu=0.32 \mathrm{~mm}^{-1}$

$T=89 \mathrm{~K}$

Rod, yellow

$0.32 \times 0.14 \times 0.14 \mathrm{~mm}$

6374 measured reflections

2233 independent reflections

1959 reflections with $I>2 \sigma(I)$

$R_{\text {int }}=0.019$

$\theta_{\max }=26.4^{\circ}, \theta_{\min }=2.3^{\circ}$

$h=-9 \rightarrow 11$

$k=-10 \rightarrow 9$

$l=-17 \rightarrow 8$ 


\section{Refinement}

Refinement on $F^{2}$

Least-squares matrix: full

$R\left[F^{2}>2 \sigma\left(F^{2}\right)\right]=0.033$

$w R\left(F^{2}\right)=0.088$

$S=1.06$

2233 reflections

163 parameters

0 restraints

Primary atom site location: structure-invariant direct methods
Secondary atom site location: difference Fourier map

Hydrogen site location: inferred from neighbouring sites

$\mathrm{H}$-atom parameters constrained

$w=1 /\left[\sigma^{2}\left(F_{\mathrm{o}}^{2}\right)+(0.0465 P)^{2}+0.6248 P\right]$ where $P=\left(F_{\mathrm{o}}{ }^{2}+2 F_{\mathrm{c}}{ }^{2}\right) / 3$

$(\Delta / \sigma)_{\max }=0.001$

$\Delta \rho_{\max }=0.28 \mathrm{e} \AA^{-3}$

$\Delta \rho_{\min }=-0.49$ e $\AA^{-3}$

Special details

Geometry. All e.s.d.'s (except the e.s.d. in the dihedral angle between two l.s. planes) are estimated using the full covariance matrix. The cell e.s.d.'s are taken into account individually in the estimation of e.s.d.'s in distances, angles and torsion angles; correlations between e.s.d.'s in cell parameters are only used when they are defined by crystal symmetry. An approximate (isotropic) treatment of cell e.s.d.'s is used for estimating e.s.d.'s involving 1.s. planes.

Refinement. Refinement of $F^{2}$ against ALL reflections. The weighted $R$-factor $w R$ and goodness of fit $S$ are based on $F^{2}$, conventional $R$-factors $R$ are based on $F$, with $F$ set to zero for negative $F^{2}$. The threshold expression of $F^{2}>\sigma\left(F^{2}\right)$ is used only for calculating $R$-factors (gt) etc. and is not relevant to the choice of reflections for refinement. $R$-factors based on $F^{2}$ are statistically about twice as large as those based on $F$, and $R$ - factors based on ALL data will be even larger.

Fractional atomic coordinates and isotropic or equivalent isotropic displacement parameters $\left(\AA^{2}\right)$

\begin{tabular}{|c|c|c|c|c|}
\hline & $x$ & $y$ & $z$ & $U_{\text {iso }} * / U_{\text {eq }}$ \\
\hline $\mathrm{S}$ & $0.28908(4)$ & $0.49088(4)$ & $0.39904(3)$ & $0.01554(13)$ \\
\hline $\mathrm{O} 1$ & $0.79782(14)$ & $0.02166(15)$ & $0.65112(9)$ & 0.0229 \\
\hline $\mathrm{O} 2$ & $0.75778(13)$ & $-0.16192(14)$ & $0.74420(9)$ & $0.0199(3)$ \\
\hline $\mathrm{O} 3$ & $0.23786(14)$ & $-0.34566(14)$ & $0.65950(9)$ & 0.0230 \\
\hline $\mathrm{O} 4$ & $0.06497(14)$ & $-0.21217(16)$ & $0.55662(12)$ & $0.0343(4)$ \\
\hline $\mathrm{O5}$ & $0.31800(13)$ & $0.32888(13)$ & $0.45017(8)$ & $0.0171(3)$ \\
\hline O6 & $0.31513(15)$ & $0.60687(14)$ & $0.47320(9)$ & 0.0259 \\
\hline O7 & $0.37522(14)$ & $0.49312(14)$ & $0.33100(9)$ & 0.0223 \\
\hline N1 & $0.71506(16)$ & $-0.05791(16)$ & $0.68364(10)$ & $0.0166(3)$ \\
\hline $\mathrm{N} 2$ & $0.19482(16)$ & $-0.23139(16)$ & $0.60944(11)$ & 0.0191 \\
\hline $\mathrm{C} 1$ & $0.35300(19)$ & $0.15179(18)$ & $0.58246(11)$ & $0.0149(3)$ \\
\hline $\mathrm{C} 2$ & $0.50631(18)$ & 0.11998 (19) & $0.61537(11)$ & $0.0155(3)$ \\
\hline $\mathrm{H} 2 \mathrm{~A}$ & 0.5761 & 0.1957 & 0.6155 & $0.019 *$ \\
\hline $\mathrm{C} 3$ & $0.55267(18)$ & $-0.02626(19)$ & $0.64782(12)$ & $0.0148(3)$ \\
\hline $\mathrm{C} 4$ & $0.45474(18)$ & $-0.14488(18)$ & $0.64856(11)$ & $0.0148(3)$ \\
\hline $\mathrm{H} 4 \mathrm{~A}$ & 0.4880 & -0.2418 & 0.6720 & $0.018 *$ \\
\hline $\mathrm{C} 5$ & 0.30408 (18) & $-0.10912(18)$ & $0.61204(11)$ & $0.0150(3)$ \\
\hline C6 & $0.25038(19)$ & 0.03509 (19) & $0.57931(12)$ & $0.0154(3)$ \\
\hline H6A & 0.1479 & 0.0536 & 0.5557 & $0.019 *$ \\
\hline $\mathrm{C} 7$ & 0.29970 (19) & $0.31110(18)$ & $0.54971(12)$ & $0.0163(3)$ \\
\hline H7A & 0.1955 & 0.3235 & 0.5470 & $0.020 *$ \\
\hline H7B & 0.3593 & 0.3862 & 0.5951 & $0.020 *$ \\
\hline $\mathrm{C} 8$ & $0.0984(2)$ & $0.4858(2)$ & $0.33155(17)$ & $0.0319(5)$ \\
\hline H8A & 0.0702 & 0.5810 & 0.2974 & $0.048 *$ \\
\hline H8B & 0.0399 & 0.4696 & 0.3760 & $0.048 *$ \\
\hline
\end{tabular}


supporting information

\begin{tabular}{|c|c|c|c|c|c|c|}
\hline $\mathrm{H} 8 \mathrm{C}$ & 0.0807 & & & 0.2843 & $0.048^{*}$ & \\
\hline \multicolumn{7}{|c|}{ Atomic displacement parameters $\left(\AA^{2}\right)$} \\
\hline & $U^{11}$ & $U^{22}$ & $U^{33}$ & $U^{12}$ & $U^{13}$ & $U^{23}$ \\
\hline$S$ & $0.0168(2)$ & $0.0125(2)$ & $0.0157(2)$ & $-0.00083(14)$ & $0.00236(16)$ & $0.00293(14)$ \\
\hline $\mathrm{O} 1$ & $0.0180(6)$ & $0.0286(7)$ & $0.0219(7)$ & $-0.0040(5)$ & $0.0057(5)$ & $0.0021(5)$ \\
\hline $\mathrm{O} 2$ & $0.0211(6)$ & $0.0168(6)$ & $0.0175(6)$ & $0.0029(5)$ & $-0.0006(5)$ & $0.0006(5)$ \\
\hline $\mathrm{O} 3$ & $0.0241(7)$ & $0.0172(6)$ & $0.0263(7)$ & $-0.0011(5)$ & $0.0057(5)$ & $0.0086(5)$ \\
\hline $\mathrm{O} 4$ & $0.0164(7)$ & $0.0248(7)$ & $0.0529(9)$ & $-0.0036(5)$ & $-0.0030(6)$ & $0.0140(7)$ \\
\hline O5 & $0.0237(6)$ & $0.0143(6)$ & $0.0134(6)$ & $0.0010(5)$ & $0.0055(5)$ & $0.0022(4)$ \\
\hline O6 & $0.0421(8)$ & $0.0133(6)$ & $0.0230(6)$ & $-0.0022(5)$ & $0.0105(6)$ & $0.0000(5)$ \\
\hline $\mathrm{O} 7$ & $0.0250(7)$ & $0.0227(7)$ & $0.0200(6)$ & $-0.0002(5)$ & $0.0079(5)$ & $0.0053(5)$ \\
\hline N1 & $0.0176(7)$ & $0.0164(7)$ & $0.0138(6)$ & $-0.0001(6)$ & $0.0018(6)$ & $-0.0035(6)$ \\
\hline $\mathrm{N} 2$ & $0.0189(7)$ & $0.0149(7)$ & $0.0227(7)$ & $-0.0007(6)$ & $0.0052(6)$ & $0.0035(6)$ \\
\hline $\mathrm{C} 1$ & $0.0207(8)$ & $0.0135(8)$ & $0.0100(7)$ & $0.0010(6)$ & $0.0038(6)$ & $0.0006(6)$ \\
\hline $\mathrm{C} 2$ & $0.0190(8)$ & $0.0147(8)$ & $0.0126(7)$ & $-0.0022(6)$ & $0.0044(6)$ & $-0.0009(6)$ \\
\hline $\mathrm{C} 3$ & $0.0155(8)$ & $0.0178(8)$ & $0.0099(7)$ & $0.0001(6)$ & $0.0018(6)$ & $-0.0016(6)$ \\
\hline $\mathrm{C} 4$ & $0.0200(8)$ & $0.0132(8)$ & $0.0101(7)$ & $0.0020(6)$ & $0.0030(6)$ & $0.0000(6)$ \\
\hline $\mathrm{C} 5$ & $0.0185(8)$ & $0.0140(8)$ & $0.0124(7)$ & $-0.0028(6)$ & $0.0046(6)$ & $-0.0004(6)$ \\
\hline C6 & $0.0171(8)$ & $0.0163(8)$ & $0.0120(7)$ & $0.0010(6)$ & $0.0031(6)$ & $0.0005(6)$ \\
\hline $\mathrm{C} 7$ & $0.0216(8)$ & $0.0136(8)$ & $0.0136(8)$ & $-0.0003(6)$ & $0.0053(6)$ & 0.0009 (6) \\
\hline $\mathrm{C} 8$ & $0.0177(9)$ & $0.0331(11)$ & $0.0390(12)$ & $0.0005(8)$ & $-0.0002(8)$ & $0.0141(9)$ \\
\hline
\end{tabular}

Geometric parameters $\left(\AA,{ }^{o}\right)$

\begin{tabular}{llll}
\hline $\mathrm{S}-\mathrm{O} 6$ & $1.4279(13)$ & $\mathrm{C} 1-\mathrm{C} 7$ & $1.507(2)$ \\
$\mathrm{S}-\mathrm{O} 7$ & $1.4290(13)$ & $\mathrm{C} 2-\mathrm{C} 3$ & $1.385(2)$ \\
$\mathrm{S}-\mathrm{O} 5$ & $1.5783(12)$ & $\mathrm{C} 2-\mathrm{H} 2 \mathrm{~A}$ & 0.9300 \\
$\mathrm{~S}-\mathrm{C} 8$ & $1.7538(19)$ & $\mathrm{C} 3-\mathrm{C} 4$ & $1.387(2)$ \\
$\mathrm{O} 1-\mathrm{N} 1$ & $1.2289(19)$ & $\mathrm{C} 4-\mathrm{C} 5$ & $1.384(2)$ \\
$\mathrm{O} 2-\mathrm{N} 1$ & $1.2322(18)$ & $\mathrm{C} 4-\mathrm{H} 4 \mathrm{~A}$ & 0.9300 \\
$\mathrm{O} 3-\mathrm{N} 2$ & $1.2223(18)$ & $\mathrm{C} 5-\mathrm{C} 6$ & $1.386(2)$ \\
$\mathrm{O} 4-\mathrm{N} 2$ & $1.2323(19)$ & $\mathrm{C} 6-\mathrm{H} 6 \mathrm{~A}$ & 0.9300 \\
$\mathrm{O} 5-\mathrm{C} 7$ & $1.4773(19)$ & $\mathrm{C} 7-\mathrm{H} 7 \mathrm{~A}$ & 0.9700 \\
$\mathrm{~N} 1-\mathrm{C} 3$ & $1.476(2)$ & $\mathrm{C} 7-\mathrm{H} 7 \mathrm{~B}$ & 0.9700 \\
$\mathrm{~N} 2-\mathrm{C} 5$ & $1.473(2)$ & $\mathrm{C} 8-\mathrm{H} 8 \mathrm{~A}$ & 0.9600 \\
$\mathrm{C} 1-\mathrm{C} 6$ & $1.394(2)$ & $\mathrm{C} 8-\mathrm{H} 8 \mathrm{~B}$ & 0.9600 \\
$\mathrm{C} 1-\mathrm{C} 2$ & $1.397(2)$ & $\mathrm{C} 8-\mathrm{H} 8 \mathrm{C}$ & 0.9600 \\
& & & $115.40(15)$ \\
$\mathrm{O} 6-\mathrm{S}-\mathrm{O} 7$ & $118.62(8)$ & $\mathrm{C} 5-\mathrm{C} 4-\mathrm{C} 3$ & 122.3 \\
$\mathrm{O} 6-\mathrm{S}-\mathrm{O} 5$ & $109.52(7)$ & $\mathrm{C} 5-\mathrm{C} 4-\mathrm{H} 4 \mathrm{~A}$ & 122.3 \\
$\mathrm{O} 7-\mathrm{S}-\mathrm{O} 5$ & $105.51(7)$ & $\mathrm{C} 3-\mathrm{C} 4-\mathrm{H} 4 \mathrm{~A}$ & $123.88(15)$ \\
$\mathrm{O} 6-\mathrm{S}-\mathrm{C} 8$ & $109.88(10)$ & $\mathrm{C} 4-\mathrm{C} 5-\mathrm{C} 6$ & $117.80(14)$ \\
$\mathrm{O} 7-\mathrm{S}-\mathrm{C} 8$ & $108.68(9)$ & $\mathrm{C} 4-\mathrm{C} 5-\mathrm{N} 2$ & $118.32(14)$ \\
$\mathrm{O} 5-\mathrm{S}-\mathrm{C} 8$ & $103.51(8)$ & $\mathrm{C} 6-\mathrm{C} 5-\mathrm{N} 2$ & $118.68(15)$ \\
$\mathrm{C} 7-\mathrm{O} 5-\mathrm{S}$ & $118.62(10)$ & $\mathrm{C} 5-\mathrm{C} 6-\mathrm{C} 1$ & 120.7 \\
$\mathrm{O} 1-\mathrm{N} 1-\mathrm{O} 2$ & $124.75(14)$ & $\mathrm{C} 5-\mathrm{C} 6-\mathrm{H} 6 \mathrm{~A}$ &
\end{tabular}




$\begin{array}{llll}\mathrm{O} 1-\mathrm{N} 1-\mathrm{C} 3 & 117.64(13) & \mathrm{C} 1-\mathrm{C} 6-\mathrm{H} 6 \mathrm{~A} & 120.7 \\ \mathrm{O} 2-\mathrm{N} 1-\mathrm{C} 3 & 117.61(14) & \mathrm{O} 5-\mathrm{C} 7-\mathrm{C} 1 & 105.58(13) \\ \mathrm{O} 3-\mathrm{N} 2-\mathrm{O} 4 & 123.85(14) & \mathrm{O} 5-\mathrm{C} 7-\mathrm{H} 7 \mathrm{~A} & 110.6 \\ \mathrm{O} 3-\mathrm{N} 2-\mathrm{C} 5 & 118.35(13) & \mathrm{C} 1-\mathrm{C} 7-\mathrm{H} 7 \mathrm{~A} & 110.6 \\ \mathrm{O} 4-\mathrm{N} 2-\mathrm{C} 5 & 117.80(14) & \mathrm{O} 5-\mathrm{C} 7-\mathrm{H} 7 \mathrm{~B} & 110.6 \\ \mathrm{C} 6-\mathrm{C} 1-\mathrm{C} 2 & 119.55(15) & \mathrm{C} 1-\mathrm{C} 7-\mathrm{H} 7 \mathrm{~B} & 110.6 \\ \mathrm{C} 6-\mathrm{C} 1-\mathrm{C} 7 & 120.50(15) & \mathrm{H} 7 \mathrm{~A}-\mathrm{C} 7-\mathrm{H} 7 \mathrm{~B} & 108.8 \\ \mathrm{C} 2-\mathrm{C} 1-\mathrm{C} 7 & 119.95(15) & \mathrm{S}-\mathrm{C} 8-\mathrm{H} 8 \mathrm{~A} & 109.5 \\ \mathrm{C} 3-\mathrm{C} 2-\mathrm{C} 1 & 118.88(15) & \mathrm{S}-\mathrm{C} 8-\mathrm{H} 8 \mathrm{~B} & 109.5 \\ \mathrm{C} 3-\mathrm{C} 2-\mathrm{H} 2 \mathrm{~A} & 120.6 & \mathrm{H} 8 \mathrm{~A}-\mathrm{C} 8-\mathrm{H} 8 \mathrm{~B} & 109.5 \\ \mathrm{C} 1-\mathrm{C} 2-\mathrm{H} 2 \mathrm{~A} & 120.6 & \mathrm{~S}-\mathrm{C} 8-\mathrm{H} 8 \mathrm{C} & 109.5 \\ \mathrm{C} 2-\mathrm{C} 3-\mathrm{C} 4 & 123.55(15) & \mathrm{H} 8 \mathrm{~A}-\mathrm{C} 8-\mathrm{H} 8 \mathrm{C} & 109.5 \\ \mathrm{C} 2-\mathrm{C} 3-\mathrm{N} 1 & 118.36(14) & \mathrm{H} 8 \mathrm{~B}-\mathrm{C} 8-\mathrm{H} 8 \mathrm{C} & 109.5 \\ \mathrm{C} 4-\mathrm{C} 3-\mathrm{N} 1 & 118.09(14) & & \end{array}$

